\title{
Higher order structure in a complex plasma
}

\author{
Z. Donkó, ${ }^{1,2}$ P. Hartmann, ${ }^{1,2}$ P. Magyar, ${ }^{1}$ G. J. Kalman, ${ }^{2}$ and K. I. Golden ${ }^{3}$ \\ ${ }^{1}$ Institute for Solid State Physics and Optics, Wigner Research Centre for Physics, Hungarian Academy of \\ Sciences, Konkoly-Thege Miklós str. 29-33, H-1121 Budapest, Hungary \\ ${ }^{2}$ Physics Department, Boston College, Chestnut Hill, Massachusetts 02467, USA \\ ${ }^{3}$ Department of Mathematics and Statistics, and Department of Physics, University of Vermont, Burlington \\ Vermont 05401, USA
}

(Received 15 June 2017; accepted 21 August 2017; published online 14 September 2017)

The direct experimental determination of the 3-point static structure function $S^{(3)}\left(\mathrm{k}_{1}, \mathrm{k}_{2}, \mathrm{k}_{0}\right)$ of a 2 dimensional dusty plasma liquid is presented. The measurements are complemented by molecular dynamics simulations of the system, using parameters (dust charge, plasma frequency, coupling and screening coefficients), which are derived from the experimentally obtained 2-point static structure function $S^{(2)}$, as well as the dynamic structure function and current-current fluctuation spectra. The experimental results of $S^{(3)}$ are in good agreement with those of the simulations, including the (low wavenumber) domain, where $S^{(3)}$ acquires negative values. The "Convolution Approximation" (giving $S^{(3)}$ in a factorized form of $S^{(2)}$ functions) clearly breaks down in this domain; however, it is found to be a useful aid for explaining the main features of the $S^{(3)}\left(\mathrm{k}_{1}, \mathrm{k}_{2}, \mathrm{k}_{0}\right)$ functions, for which (experimental and simulation) maps are presented at selected values of one of its arguments. Published by AIP Publishing. [http://dx.doi.org/10.1063/1.4990510]

\section{INTRODUCTION}

Correlation functions are centrally important for the characterization of liquid-phase materials. ${ }^{1,2}$ While in most studies, only the 2-particle (pair) correlation function $g^{(2)}(r)$ is addressed, it is understood that complete characterization of the systems can only be achieved if the hierarchy of $n$-particle correlation functions is considered. ${ }^{3}$ While $g^{(2)}(r)$ - the lowest order member of this hierarchy - gives the probability of finding particles at a given distance from each other, the next member, the 3-particle or "triplet" correlation function $g^{(3)}$, e.g., expresses the probability of finding 3 particles at given mutual separations from each other, thereby providing additional structural information beyond $g^{(2)}$, about the local orientational ordering of the particles.

The real-space correlation functions are linked with wavenumber-space static structure functions, $S^{(n)}$, that build a hierarchy ${ }^{4}$ as well, similarly to the $g^{(n)}$ real-space correlation functions. The importance of this hierarchy of the structure functions is highlighted by the fact that they are linked via the Fluctuation-Dissipation Theorem $(\mathrm{FDT})^{5}$ with the response of many-body systems to external, e.g., potential, perturbations. The 2-point structure function $S^{(2)}$ is related to the linear response, while, in a similar fashion, higher-order structure functions are linked via the higher-order FDT with the nonlinear response of these systems. ${ }^{6-9}$

The correlation functions and the static structure functions can readily be determined if the coordinates of the particles comprising the system are known. The real space functions $g^{(2)}$ and $g^{(3)}$ can be constructed, respectively, by counting particle pairs separated by certain distances, and by counting triplets of particles with certain mutual distances. The two lowest order (and most relevant) structure functions can be computed based on the definitions

$$
\begin{aligned}
S^{(2)}(\mathbf{k}) & =\frac{1}{N}\langle n(\mathbf{k}, t) n(-\mathbf{k}, t)\rangle, \\
S^{(3)}\left(\mathbf{k}_{1}, \mathbf{k}_{2}, \mathbf{k}_{0}\right) & =\frac{1}{N}\left\langle n\left(\mathbf{k}_{1}, t\right) n\left(\mathbf{k}_{2}, t\right) n\left(\mathbf{k}_{0}, t\right)\right\rangle,
\end{aligned}
$$

where $N$ is the number of particles, $\mathbf{k}_{1}+\mathbf{k}_{2}+\mathbf{k}_{0}=0, S^{(3)}$ is a symmetric function of these arguments, and $n(\mathbf{k}, t)$ is the microscopic density in Fourier space defined as

$$
n(\mathbf{k}, t)=\sum_{j=1}^{N} \exp \left[-i \mathbf{k} \cdot \mathbf{r}_{j}(t)\right]
$$

In isotropic liquids, $S^{(2)}(k)$ depends on a single scalar argument, similarly to its real-space counterpart, the $g^{(2)}(r)$ pair correlation function.

We note that the static structure functions have their dynamic counterparts. The (2-point) dynamic structure function $S(\mathbf{k}, \omega)$ is the lowest order member of this hierarchy.

Returning to the static properties, the evaluation of the above formulae for the structure functions is straightforward in simulations, but their use in experiments is restricted to a few, rather exceptional physical systems where the particle coordinates can be directly measured. Two notable types of such systems are colloidal suspensions and dusty plasmas, in which the characteristic time and length scales allow tracing the individual charged microscopic particles. ${ }^{10}$ For the vast majority of physical systems (materials), the coordinates of the individual particles, however, cannot be directly measured. In such cases, experimental results of $g^{(2)}(r)$ are usually obtained via measurement of the static structure function $S^{(2)}(k)$ using (electron, neutron, or x-ray) scattering methods ${ }^{11-23}$ and utilizing the Fourier transform relationship between $g^{(2)}(r)$ and $S^{(2)}(k)$.

The direct determination of higher-order correlation functions is virtually impossible for such conventional 
materials. Information about the 3-particle correlation function, $g^{(3)}$, can, nonetheless be inferred in an indirect way, ${ }^{1,2,24}$ from measurements of the two-particle quantities at a series of pressure values at constant system temperature and using the relation between the pressure derivative of the (2-particle) pair correlation function $\left(\partial g^{(2)} / \partial p\right)_{T}$ and $g^{(3)}$.

An often used approximate form for the 3-particle correlation function is given as

$$
g_{\mathrm{SA}}^{(3)}\left(\mathbf{r}_{1}, \mathbf{r}_{2}, \mathbf{r}_{3}\right)=g^{(2)}\left(\mathbf{r}_{1}-\mathbf{r}_{2}\right) g^{(2)}\left(\mathbf{r}_{2}-\mathbf{r}_{3}\right) g^{(2)}\left(\mathbf{r}_{3}-\mathbf{r}_{1}\right),
$$

which is called the "superposition approximation" (SA), or "Kirkwood approximation." The performance of the SA has been tested for several types of systems using different simulation approaches. Hard sphere and hard disk systems, e.g., were examined using Monte Carlo simulations, ${ }^{25}$ while Reverse Monte Carlo and Molecular Dynamics (MD) methods have been applied ${ }^{26}$ for the case of liquid water. The validity of the SA was tested for binary mixtures of particles interacting via the Kob-Andersen Lennard-Jones potential and the Weeks-Chandler-Andersen potential, ${ }^{27}$ and its ability to characterize the structure of gaseous He at low temperatures was also checked. ${ }^{28}$ For review, see Ref. 29.

The colloidal and dusty plasma systems, already mentioned above, provide unique possibilities for checking the accuracy of the SA as the particle correlation and structure functions can readily be obtained from measured particle coordinates in these systems. In Ref. 30, videomicroscopy was used to follow the trajectories of magnetized colloidal grains floating on the water-air interface, i.e., forming a twodimensional system. The structure of the 3-particle correlation function $g^{(3)}$ was studied, in particular, the limitation of the superposition approximation has been tested. The (exact) Born-Green equation ${ }^{31}$ was found to be satisfied, but the 3particle correlation function computed from the SA showed significant disagreement at high $\Gamma$ values with the measured $g^{(3)}$.

Experimental and simulation studies of the validity of the SA, and in more general, three-particle correlations in a two-dimensional dusty plasma layer have been presented in Ref. 32. The $g^{(3)}$ functions derived from the experimental data were found to reflect the six-fold symmetry of the particles' arrangement for most conditions and confirmed the emergence of the onset of orientational ordering of the particles towards the freezing point. It was found that with increasing coupling new peaks at multiples of the most probable pair separation, $r^{*}$, appear in the measured $g^{(3)}$, indicating the extension of the correlation length. Another investigation, ${ }^{33}$ utilizing a similar experimental system, has examined the range of validity of integral equation approaches of statistical physics, like the Percus-Yevick relation and the Hypernetted Chain Approximation, which allow computation of correlation functions of isotropic liquids. The behavior of the 3-particle correlation function $g^{(3)}$ was also addressed in an investigation of the melting of $2 \mathrm{D}$ dust crystals ${ }^{34}$ and in studies of phenomena taking place in 2D Coulomb clusters. ${ }^{35}$

Besides investigations in real space, several studies ${ }^{36-39}$ (including our previous work $^{40}$ ) have addressed the properties of the 3-point structure function $S^{(3)}\left(\mathbf{k}_{1}, \mathbf{k}_{2}, \mathbf{k}_{0}\right)$ and the validity of the factorization in $\mathbf{k}$-space, or the socalled "Convolution Approximation" (CA)

$$
S^{(3)}\left(\mathbf{k}_{1}, \mathbf{k}_{2}, \mathbf{k}_{0}\right) \cong S^{(2)}\left(\mathbf{k}_{1}\right) S^{(2)}\left(\mathbf{k}_{2}\right) S^{(2)}\left(\mathbf{k}_{0}\right),
$$

which is related to the exact $S^{(3)}\left(\mathbf{k}_{1}, \mathbf{k}_{2}, \mathbf{k}_{0}\right)$ by

$$
\begin{aligned}
S^{(3)}\left(\mathbf{k}_{1}, \mathbf{k}_{2}, \mathbf{k}_{0}\right)= & S^{(2)}\left(\mathbf{k}_{1}\right) S^{(2)}\left(\mathbf{k}_{2}\right) S^{(2)}\left(\mathbf{k}_{0}\right) \\
& \times\left[1+n_{0}^{2} c^{(3)}\left(\mathbf{k}_{1}, \mathbf{k}_{2}, \mathbf{k}_{0}\right)\right],
\end{aligned}
$$

where $c^{(3)}\left(\mathbf{k}_{1}, \mathbf{k}_{2}, \mathbf{k}_{0}\right)$ is the 3-point direct correlation function. ${ }^{41}$ Obviously, the CA corresponds to setting $c^{(3)}$ to zero. Here, $n_{0}$ is the number density of particles and $\mathbf{k}_{1}+\mathbf{k}_{2}$ $+\mathbf{k}_{0}=0$. The implication of the CA concerning the resulting structure of $g^{(3)}$ was discussed in Ref. 40, where it was also shown via Molecular Dynamics simulations that the quadratic response of the system (linked with $S^{(3)}$ via the quadratic FDT) changes sign at the point when the term $1+n_{0}^{2} c^{(3)}\left(\mathbf{k}_{1}, \mathbf{k}_{2}\right)$ in (6) changes sign, which clearly cannot be accounted for by the CA as given by (5), since $S^{(2)}$ is positive definite.

In this work, we report the (to the best of our knowledge) first direct experimental measurement of the 3-point static structure function $S^{(3)}$ of a two-dimensional dusty plasma liquid and the experimental observation of the violation of the "Convolution Approximation." The experimental determination of $S^{(3)}$ is made possible by realizing very stable experimental conditions and by the recording and analysis of extensive amounts of data. The experimental studies are accompanied by Molecular Dynamics simulations, which (i) assist the determination of the main parameters of the dust system and (ii) make it possible to derive "exact" $S^{(3)}$ data as well, which are being compared with their experimental counterparts. The details of the experiments and the simulations are given in Sec. II. The determination of the main plasma characteristics and the experimental and simulation results of $S^{(3)}$ are presented and compared to each other in Sec. III. Section IV gives a short summary of the work.

\section{METHODS}

\section{A. Experimental realization of the $2 \mathrm{D}$ dusty plasma layer}

The physical setting considered here is a many-body system composed of charged dust particles that settle in a (nearly-)two-dimensional layer in the experimental system described below. The dust particles acquire a high negative charge due to the flow of electrons and ions from the surrounding weakly ionized plasma medium. The electrostatic interaction between the particles is screened by the plasma, and can thus be characterized by a Debye-Hückel or Yukawa potential

$$
\phi(r)=\frac{Q \exp \left(-r / \lambda_{\mathrm{D}}\right)}{4 \pi \varepsilon_{0} r},
$$

where $Q$ is the charge of the particles and $\lambda_{\mathrm{D}}$ is the screening (Debye) length that depends on the plasma properties. The 
ratio of the inter-particle potential energy to the thermal energy is expressed by the coupling parameter

$$
\Gamma=\frac{Q^{2}}{4 \pi \varepsilon_{0} a k_{\mathrm{B}} T},
$$

where $T$ is the (dust) temperature, $a=\left(\pi n_{0}\right)^{-1 / 2}$ is the Wigner-Seitz radius, and $n_{0}$ is the areal number density of particles. The strength of screening of the Coulomb interaction is expressed by the parameter

$$
\kappa=a / \lambda_{\mathrm{D}}
$$

The two dimensionless parameters $\Gamma$ and $\kappa$ characterize the dust system completely in the framework of the Yukawa model. An additional important parameter is the nominal plasma frequency

$$
\omega_{0}=\sqrt{n Q^{2} / \varepsilon_{0} M},
$$

that governs the dynamics of the system. Here, $\varepsilon_{0}$ is the permittivity of free space and $M$ is the mass of the particles.

The experimental setup is shown in Fig. 1 and is briefly described below (for more details, see Ref. 43). A lowionization degree plasma in argon gas (at a pressure of $\sim 1 \mathrm{~Pa}$ ) is created between two flat disk electrodes of $18 \mathrm{~cm}$ diameter, separated by a distance of $13 \mathrm{~cm}$, using radio frequency excitation at $13.56 \mathrm{MHz}$, at a power level of $\sim 10 \mathrm{~W}$.

Several thousand melamine-formaldehyde particles, with a diameter of $d=4.38 \mu \mathrm{m}( \pm 1 \%)$ are dispersed into the plasma via an opening of the upper electrode. These particles acquire a negative charge in the plasma environment that typically amounts to several thousands of elementary charges. The balance of "vertical" forces acting on the particles (among which gravity and the electrostatic force originating from the electric field in the sheath that forms near the powered electrode are the dominant ones) forms a single layer. The large electric charge of the particles results in a high coupling coefficient $\left(\Gamma \propto Q^{2}\right)$, i.e., the inter-particle potential energy largely dominates over the kinetic energy of the particles. As a consequence of this strong interaction (strong "horizontal" forces) single-layer dusty plasmas typically

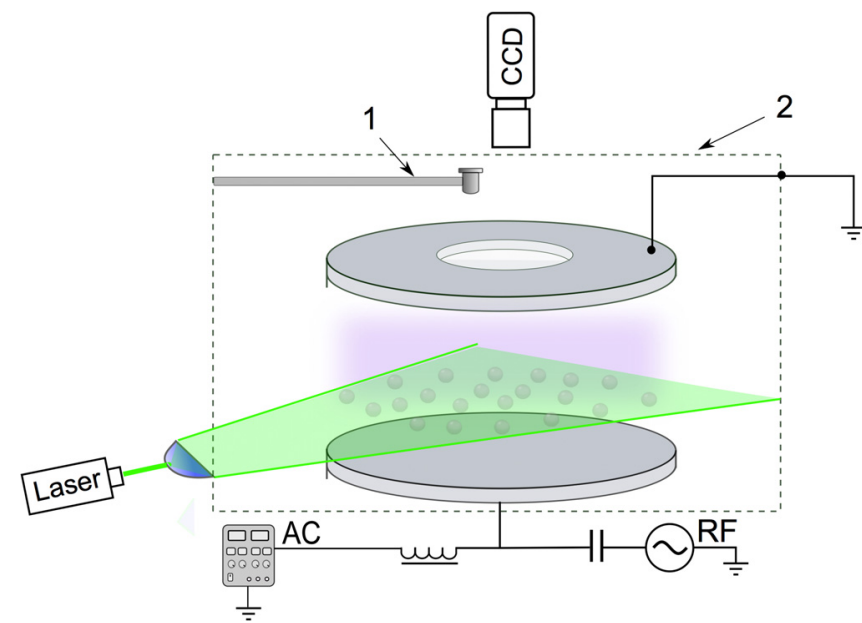

FIG. 1. Scheme of the experimental setup. 1: Particle dispenser; 2: Vacuum chamber. exhibit a crystallized structure that consists of a few large domains with different orientations ("plasma crystals"). ${ }^{42}$ In order to carry out experiments in the liquid phase of the dust system, which is our preferred setting, an additional low frequency modulation $\left(f_{\mathrm{m}}=26 \mathrm{~Hz}\right)$ is applied at the powered electrode. This modulation frequency is near the resonance frequency of the "out-of-plane" mode of the particle layer; this way, it can couple energy to the many-body system. ${ }^{44}$

The typical length and time scales of the system allow recording the evolution of the trajectories of the particles. For this, images of the dust layer, illuminated from the side with an expanded beam of a $0.5 \mathrm{~W}$ power, $440 \mathrm{~nm}$ wavelength laser, are taken using an Allied Vision Prosilica GX1050 camera with 1 MPixel resolution, at a frame rate of $100 \mathrm{~s}^{-1}$.

While our main interest lies in the investigation of the 3-point static structure function, the 2-point static and dynamic structure functions, $S^{(2)}(k)$ and $S^{(2)}(k, \omega)$, as well as the related longitudinal and transverse current correlation functions $\left[L^{(2)}(k, \omega)\right.$ and $T^{(2)}(k, \omega)$, respectively] also need to be determined to deduce the main parameters $(\Gamma$ and $\kappa)$ of the experimental system. ${ }^{45,46}$ Note that the wavenumber is used here as a scalar quantity due to the isotropy of the system in the liquid phase.

In the course of the measurements, the positions of $\approx 1200$ particles in the field of view of the camera are recorded. The 2point static and dynamic functions mentioned above can be determined from a limited set of data with high accuracy. The construction of the 3-point static structure function, based on definition (6), with a good signal to noise ratio, on the other hand, requires recording a large number of images, 600 000 in our case, generating $\sim 0.6 \mathrm{~TB}$ of data. In order to reduce the statistical and systematic noise, a 2D spatial Blackman-Nuttall window function is applied and averaging over 60 different directions of the wave-number vector $\mathbf{k}$ is performed. Images of the dust layer are processed for the particle positions with a sub-pixel resolution. ${ }^{47}$ Collection of the above number of images required continuous data recording and maintenance of stable experimental conditions for over one hour of time.

\section{B. Simulations}

Parallel to the experiments, molecular dynamics (MD) simulations are also conducted to derive the static and dynamic structure functions. We apply a standard MD method to describe the motion of $N=4000$ particles, within a square box with periodic boundary conditions, via the integration of their Newtonian equations of motion. The use of a low buffer gas pressure justifies neglecting the friction force on the dust particles. The spatial decay of the Yukawa interaction makes it possible to introduce a cutoff distance $r_{\mathrm{c}}$ in the calculation of the forces acting on the particles, beyond which the interaction of particle pairs can be neglected. Time integration is performed using the velocity-Verlet scheme.

At the initialization of the simulation runs, the positions of the particles are set randomly, while their initial velocities are sampled from a Maxwellian distribution corresponding to a specified system temperature. The simulations start with a thermalization phase, during which the particle velocities are re-scaled in each time step, in order to reach the desired 
temperature (or $\Gamma$ ). This procedure is terminated before the data collection takes place, during which the stability of the simulation is confirmed by monitoring the temperature as a function of time.

During the simulations, data for $n(\mathbf{k}, t)$ for the calculation of the static and dynamic properties are collected for specified sets of wavenumber $\mathbf{k}$. The calculation of static quantities requires a summation and averaging of these data over time; the computation of the dynamic structure function requires an additional (discrete) Fourier transform in time. ${ }^{48}$

\section{RESULTS}

\section{A. Determination of the system parameters}

The determination of the main parameters of the dusty plasma, the coupling coefficient $\Gamma$, the screening parameter $\kappa$, and the plasma frequency $\omega_{0}$ proceeds in several steps, as explained below. In the first step, the 2-point dynamic structure function is computed from the experimental data for the particle positions as

$$
S(k, \omega)=\frac{1}{2 \pi N} \lim _{\Delta T \rightarrow \infty} \frac{1}{\Delta T}|n(k, \omega)|^{2},
$$

where $\Delta T$ is the length of the data recording period and $n(k, \omega)=\mathcal{F}[n(k, t)]$ is the Fourier transforms of (3). In a similar manner, the spectrum of the transverse current correlations, $T(k, \omega)$, is obtained from the Fourier transform of the microscopic transverse current, as

$$
\tau(k, t)=\sum_{j} v_{j y}(t) \exp \left[i k x_{j}(t)\right] .
$$

Figure 2(a) shows a color map plot of the $S(k, \omega)$ spectrum as determined in the experiment. The horizontal patches at $\omega=66.5 \mathrm{rad} / \mathrm{s}$ and the vertical features at low wave-numbers in the plot originate from the modulation voltage used for melting the dust layer.

Having determined $S(k, \omega)$ and $T(k, \omega)$, the $\omega_{\mathrm{L}, \mathrm{T}}(k)$ dispersion relations of the longitudinal and transverse collective excitations can readily be obtained (here, $\omega_{\mathrm{L}, \mathrm{T}}$ are the frequencies of the two modes). As an example, the $\omega_{\mathrm{L}}(k)$ dispersion relation is overlayed as a black solid line on the plot of $S(k, \omega)$ in Fig. 2(a).

The sound speeds of these modes are given as the low- $k$ limit of the slopes of the dispersion relations, i.e., $s_{\mathrm{L}, \mathrm{T}}$ $=\lim _{k \rightarrow 0}\left(\mathrm{~d} \omega_{\mathrm{L}, \mathrm{T}} / \mathrm{d} k\right)$. The $s_{\mathrm{L}} / s_{\mathrm{T}}$ ratio is known to depend sensitively on $\kappa$, providing a way for its determination. ${ }^{48}$ Our analysis resulted in $\kappa=0.7$. As the next step, we use the property of $S(k, \omega)$ that its "plateau frequency" depends sensitively on $\kappa$, but is rather insensitive to $\Gamma$. [The "plateau frequency" is indicated in Figs. 2(a) and 2(b) by dashed white lines.] Using this relationship, we obtained $\omega_{0}=97$ $( \pm 10 \%) \mathrm{rad} / \mathrm{s}$ for the plasma frequency $(10){ }^{49}$

When $\omega_{0}$ is known, the charge of the particles can be determined, which resulted in $Q \cong 4100( \pm 20 \%) e$. This value and the WS radius $a$ determined from the particle snapshots, as $230( \pm 2 \%) \mu \mathrm{m}$ define the coupling coefficient $\Gamma$. A further $(\sim 10 \%)$ refinement of $\Gamma$ was accomplished by
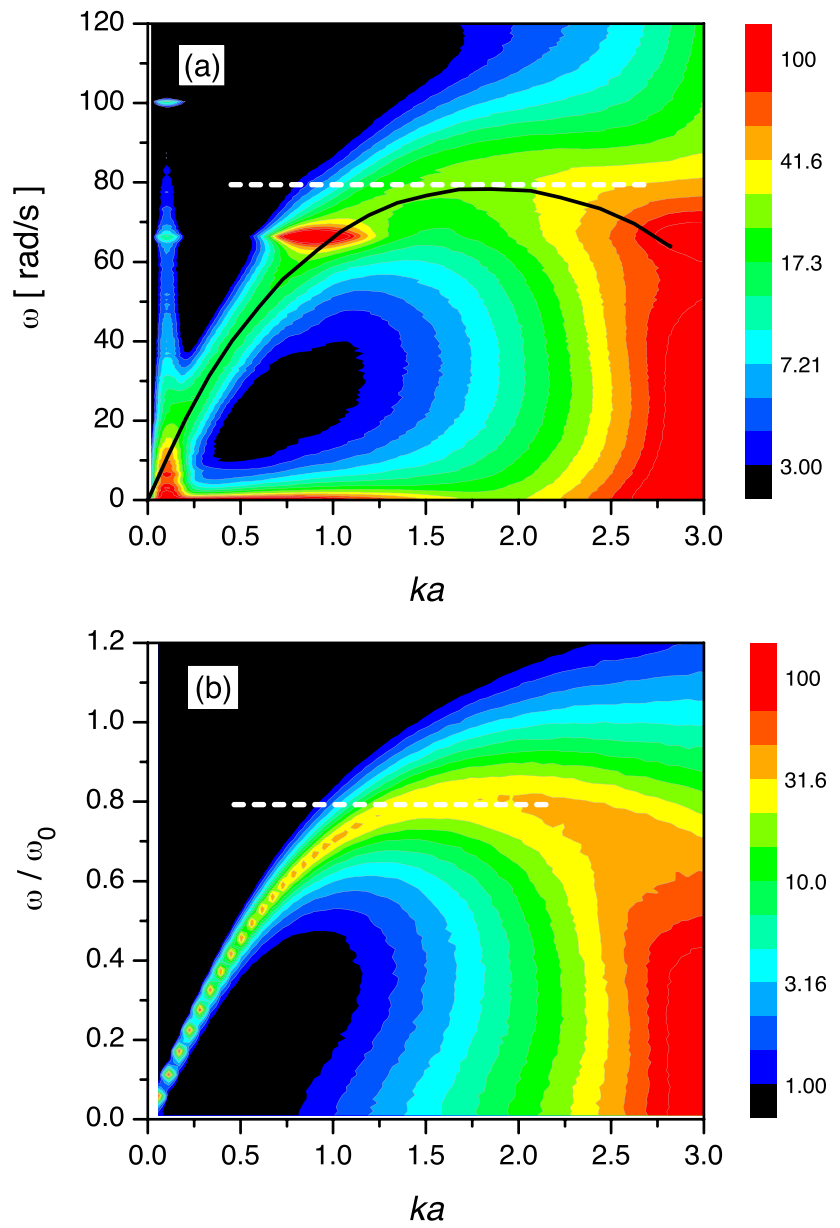

FIG. 2. Measured (a) and calculated (b) dynamic structure function $S^{(2)}(\mathbf{k}, \omega)$ of the dusty plasma layer. The black solid line in (a) marks the dispersion relation of the longitudinal mode, while the horizontal dashed white lines mark the "plateau frequency." The MD simulation uses $\Gamma=95$ and $\kappa=0.7$.

making use of the dependence ${ }^{50}$ of the static $S^{(2)}(k)$ on $\Gamma$ and $\kappa$; this procedure resulted in $\Gamma=95$.

The 2-point static structure function generated from the experimental data [via Eq. (1)] and from the MD simulations is displayed in Fig. 3. A very good agreement (both in terms

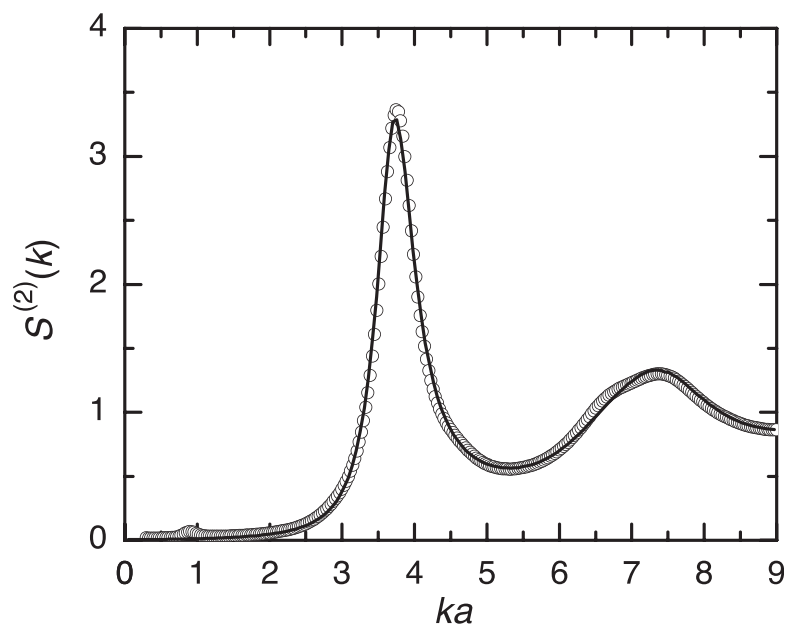

FIG. 3. Measured (open circles) and calculated (solid line) 2-point static structure function $S(k)$ of the dusty plasma layer. The MD simulation uses $\Gamma=95$ and $\kappa=0.7$ 
of the peak amplitudes and peak positions) between the two curves is found at $\Gamma=95$ and $\kappa=0.7$ values.

\section{B. Properties of the triplet static structure function}

The experimental data for the particle positions allow the determination of the 3-point $S^{(3)}\left(\mathbf{k}_{1}, \mathbf{k}_{2}, \mathbf{k}_{0}\right)$ function [via Eq. (2)] for any arguments, and-having determined the main parameters of the experimental system-the same functions can also be generated from MD simulations using the same formula. The very good signal to noise ratio of the $S^{(3)}\left(\mathbf{k}_{1}, \mathbf{k}_{2}\right.$, $\mathbf{k}_{0}$ ) functions is the outcome of the very high number of particle snapshots recorded experimentally and the consequent data analysis using several independent directions of $\mathbf{k}$, as well as of the extensive MD computations.

First, we illustrate the behavior of $S^{(3)}$ in the "diagonal direction," i.e., at equal arguments, $\mathbf{k}_{1}=\mathbf{k}_{2}$. Figure 4 shows the experimental and simulation results. (The MD simulation uses $\Gamma=95$ and $\kappa=0.7$.) A rather intriguing feature of $S^{(3)}(k, k)$ is its negative value at small wavenumbers and a negative peak at $k a \approx 1.8$. This experimentally observed feature, as well as the whole function is very well reproduced in the simulations (except for a modest difference between the peak amplitudes). This rather peculiar behavior of $S^{(3)}$ has already been noted in our previous work. The negative values of $S^{(3)}$ are, in fact, the consequence of the negative compressibility of the system. At $k$ $\rightarrow 0$, the connection between the 3-point and 2-point structure functions is $S^{(3)}\left(k_{1} \rightarrow 0, k_{2} \rightarrow 0, k_{0} \rightarrow 0\right)=\beta K\left[1-\left(n_{0} / K\right)\right.$ $\left.\frac{\mathrm{d} K}{\mathrm{~d} n_{0}}\right] S^{(2)}\left(k_{1} \rightarrow 0\right) S^{(2)}\left(k_{2} \rightarrow 0\right) S^{(2)}\left(k_{0} \rightarrow 0\right)$, where $n_{0}$ is the density and $K$ is the compressibility. Since $S^{(2)}(k \rightarrow 0)$ is positive and the term $1-\left(n_{0} / K\right) \frac{\mathrm{d} K}{\mathrm{~d} n_{0}}$ is also expected to be positive for any reasonable equation of state, negative $S^{(3)}$ values result from a negative $K$.

The main results of our study are illustrated in Fig. 5. Here, we display maps over the $\mathbf{k}_{2}$ plane of the full $S^{(3)}\left(\mathbf{k}_{1}\right.$, $\mathbf{k}_{2}, \mathbf{k}_{0}$ ) function at a few selected values of $\mathbf{k}_{1} a=\left(k_{1 x}, 0\right) a$. The plots in the first column originate from the experiment, the plots in the second column are the result of the MD

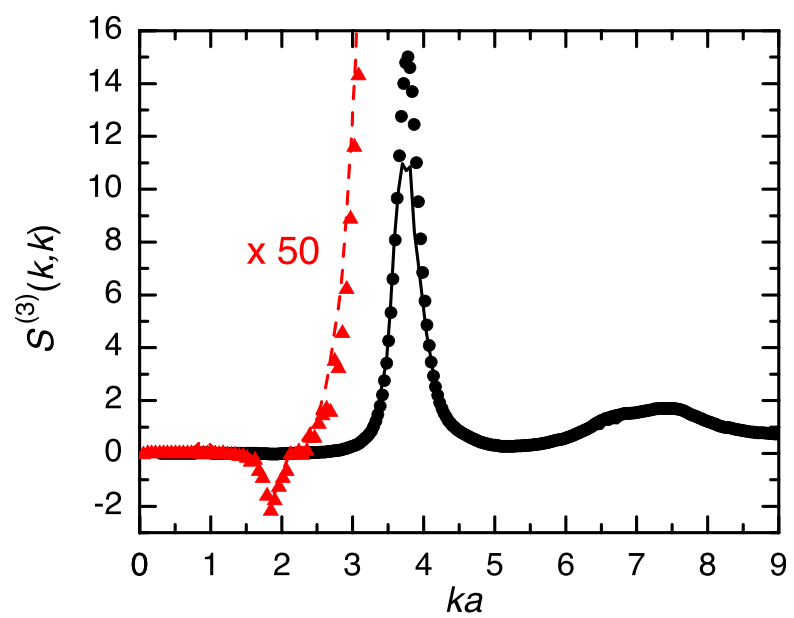

FIG. 4. Measured (filled circles) and calculated (solid line) 3-point static structure function $S^{(3)}(k, k)$ of the dusty plasma layer. The filled triangles and the dashed line represent data $50 \times$ magnified with respect to the original values, to make the negative values of the functions visible at low wavenumbers. The MD simulation uses $\Gamma=95$ and $\kappa=0.7$. simulations, while the plots in the third column have been generated from the simulation data using the Convolution Approximation (5). In order to understand the details of $S^{(3)}\left(\mathbf{k}_{1}, \mathbf{k}_{2}, \mathbf{k}_{0}\right)$ maps that originate from true 3-particle correlations, it is first useful to see what features can be attributed to the Convolution Approximation as shown in column 3 of Fig. 5.

- Since for each of the plots, $\mathbf{k}_{1}=\left(k_{1 x}, 0\right)$ is fixed and $\mathbf{k}_{2}=$ $\left(k_{2 x}, k_{2 y}\right)$, the CA takes the form

$$
S_{\mathrm{CA}}^{(3)}\left(\mathbf{k}_{1}, \mathbf{k}_{2}, \mathbf{k}_{0}\right)=S\left(k_{1}\right) S\left(k_{2}\right) S\left(\sqrt{\left(k_{1 x}+k_{2 x}\right)^{2}+k_{2 y}^{2}}\right),
$$

where the first term on the right hand side is constant. Here, and in the sequel, we drop the superscript "(2)" as the identification of the 2-point structure functions. The locus of the maxima of this function on the $\mathbf{k}_{2}$-plane traces two overlapping circles, one centered around the origin, and the other around $\mathbf{k}_{1}$. Focusing on the first one, its lefthand-side is found to be dimmed in comparison to its right-hand-side, because it overlays a low value portion of $S\left(k_{0}\right)$. A similar consideration explains the asymmetry of the other circle. In contrast, the intersection of the two circles corresponds to an enhanced intensity region,

- $S^{(3)}$ has a mirror symmetry with respect to the vertical line at $k_{2 x}=-k_{1 x} / 2$,

- High intensity peaks in the maps occur, as already noted, at intersections when the arguments of $S\left(k_{2}\right)$ and $S\left(k_{0}\right)$ correspond to $k$ values of the peaks of $S(k)$, especially to the position of the first peak, at $k=k^{*}$.

Now, looking at the results of the experiment (column 1 of Fig. 5) and of the full simulation (column 2 of Fig. 5), we see that the main features of the $\mathrm{CA}$ are recognizable. There are, however, some important deviations, revealing the working of genuine 3-particle correlations.

The first row of Fig. 5 corresponds to $\mathbf{k}_{1} a=(1.85,0)$, i.e., $k_{1}$ is chosen to be $k^{*} / 2$, where $k^{*}$ is the position of the first peak of $S^{(2)}$ (see Fig. 3). The $S^{(3)}\left(\mathbf{k}_{1}, \mathbf{k}_{2}, \mathbf{k}_{0}\right)$ function exhibits a strong maximum that shows up as a ring-like feature in red color in Fig. 5(a). This feature can be recognized as the central oval in panel (c), with the difference that the intensity distribution is fairly isotropic, indicating that the variation of intensity in $S(k)$ has little impact on $S^{(3)}$. An important novel feature is the already discussed negative peak, showing up, as another elongated ring in green/blue contour, at smaller wavenumbers. Both of these features are very well reproduced by the simulation data in Fig. 5(b).

The second value of $\mathbf{k}_{1} a=(3.76,0)$ [for which maps of $S^{(3)}\left(\mathbf{k}_{1}, \mathbf{k}_{2}, \mathbf{k}_{0}\right)$ are presented in the second row of Fig. 5] corresponds to the position of the peak of the 2-point $S(k)$, i.e., $k_{1}=k^{*}$. One can observe a similar correspondence between the ovals in panel (d) and panel (e), on the one hand, and in the CA structure in panel (f), on the other. Parts of these ring-like features are well seen (and are marked with arrows " $A$ ") in the plot of $S_{\mathrm{CA}}^{(3)}$ in Fig. 5(f). The two strongest peaks, which occur when the above two conditions are simultaneously met, at $k_{2 x}=-k^{*} / 2=-1.85 / a$ and $k_{2 y}= \pm k^{*} \sqrt{3} / 2 \cong \pm 3.20 / a$, are 

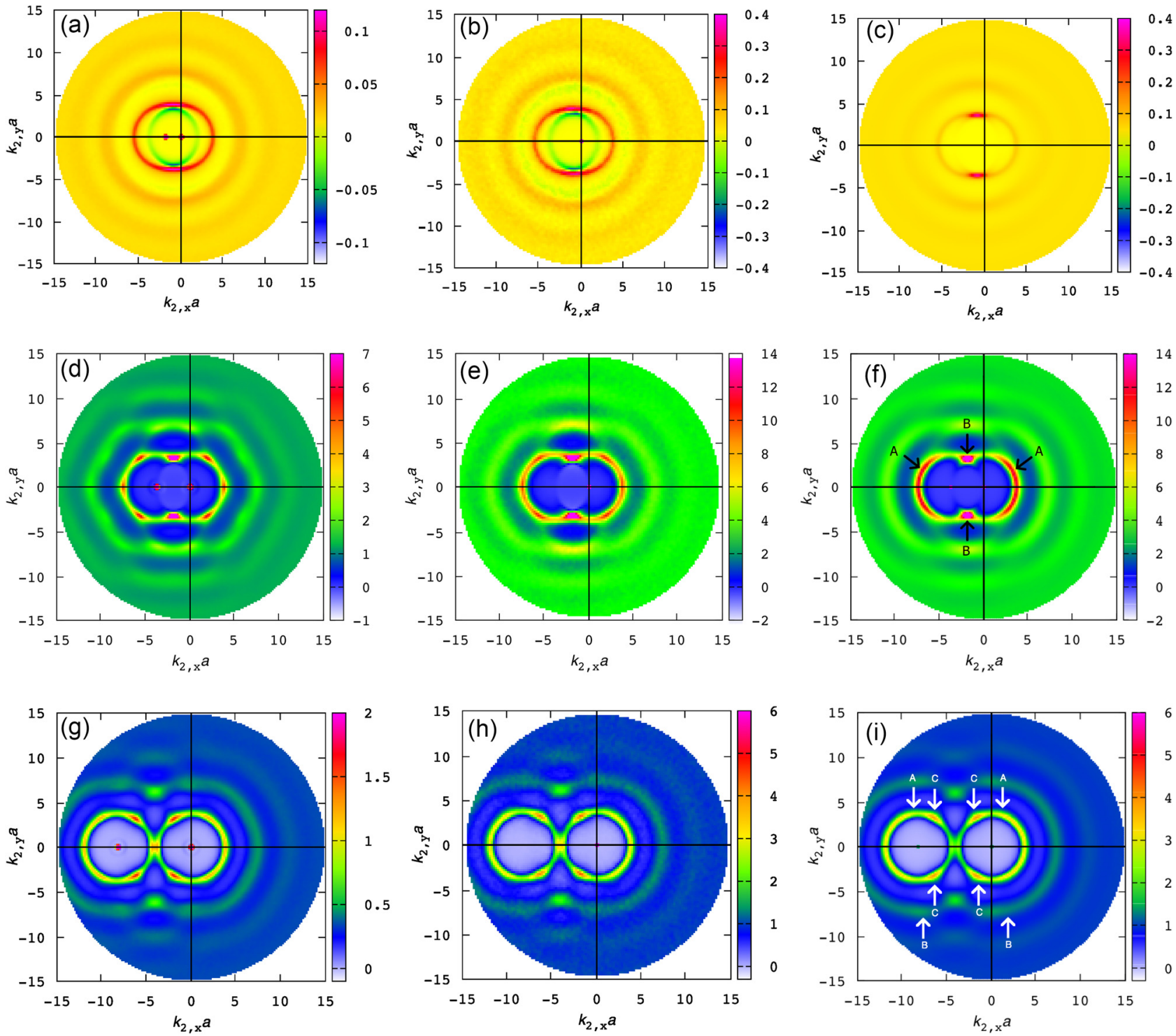

FIG. 5. Maps of the full $S^{(3)}\left(\mathbf{k}_{1}, \mathbf{k}_{2}, \mathbf{k}_{0}\right)$ at selected values of $\mathbf{k}_{1}$ : (a)-(c) $\mathbf{k}_{1} a=(1.85,0)$, (d)-(f) $\mathbf{k}_{1} a=(3.76,0)$, and (g)-(i) $\mathbf{k}_{1} a=(8.24,0)$, obtained from the experiments (first column), from the MD simulations (second column), and from the Convolution Approximation using the MD data (third column).

marked with arrows "B". Most importantly, the six-fold symmetry of the experimental arrangement of the particles is reflected in Figs. 5(d) and 5(f). Note that even though the system is in the liquid phase, with unbroken symmetry, the underlying structure of the triangular lattice, characteristic for the solid phase, shows up in the 3-particle correlations. The slight differences between the experimental and simulation results may be attributed to the remaining uncertainties in the determination of the parameters of the experimental system and its consequent imperfect reproduction by the simulations.

In the third row of Fig. 5, maps of $S^{(3)}\left(\mathbf{k}_{1}, \mathbf{k}_{2}, \mathbf{k}_{0}\right)$ for the third value of $\mathbf{k}_{1} a=(8.24,0)$ are plotted. This value was chosen according to $k_{1}=2.2 k^{*}$. In agreement with the arguments given for the previous case, peaks of the $S_{\mathrm{CA}}^{(3)}$ function are expected to appear when $k_{2}=k^{*}$ or/and $\left(2.2 k^{*}+k_{2 x}\right)^{2}$ $+k_{2 y}^{2}=k^{* 2}$. Due to the high value of $\mathbf{k}_{1}$, the oval separates into two circles. The first condition, as before, corresponds to a circle around $\left(k_{2 x}, k_{2 y}\right)=(0,0)$, while the second corresponds to a circle around $\left(k_{2 x}, k_{2 y}\right)=\left(-2.2 k^{*}, 0\right)$ (again, both circles have a radius $k^{*}$ ). Both of these circles are well visible in Fig. 5(i), where they are marked with arrows "A." The two conditions are simultaneously fulfilled at $k_{2 x}=-1.1 k^{*}$ $=-4.14 / a$ and $k_{2 y} \cong 0$, where the two curves touch. The second pair of ring-like features, concentric to these "primary" circles, is created by the second peak of $S(k)$, at $k_{2} \cong 2 k^{*}$ these features are marked with arrows "B" in Fig. 5(i). Where these rings with radii $\cong 2 k^{*}$ intersect the primary circles, additional peaks of $S_{\mathrm{CA}}^{(3)}$, marked with arrows "C," are generated. The measured and computed maps in this case are rather similar to the one obtained via the CA, although they show slightly more enhanced structures.

\section{SUMMARY}

Here, we have demonstrated that the accurate experimental determination of the 3-point static structure function $S^{(3)}\left(\mathbf{k}_{1}, \mathbf{k}_{2}, \mathbf{k}_{0}\right)$ of a two-dimensional liquid-phase dusty plasma layer is possible by establishing stable experimental conditions. We have captured a high number of images ( $\sim 600000)$ of the many-particle system during a time period 
of $\sim 1 \mathrm{~h}$. The recorded images allowed the computation of $S^{(3)}\left(\mathbf{k}_{1}, \mathbf{k}_{2}, \mathbf{k}_{0}\right)$ for arbitrary arguments. As far as many-body systems are concerned, the present experiments are among the few so far, which have resulted in direct, measured data for this 3-point structure function.

The parameters of the experimental system have been determined by a careful data analysis: the 2-point static structure function and the current-current fluctuation spectra-which were also derived from the particle snapshotsallowed the extraction of $\Gamma, \kappa$, and $\omega_{0}$. While the CA is intrinsically rotationally symmetric, this symmetry is broken in our strongly coupled system, and the six-fold symmetry of the underlying lattice structure emerges in the "exact" MD simulation results. This behavior was also recognized in the experimental results, providing further assurance on the high quality of the experimental data.

The MD computations for $S^{(3)}\left(\mathbf{k}_{1}, \mathbf{k}_{2}, \mathbf{k}_{0}\right)$, in which the above parameters were used, have generated results in very good agreement with the functions determined experimentally. Comparing with the Convolution Approximation, which gives $S^{(3)}\left(\mathbf{k}_{1}, \mathbf{k}_{2}, \mathbf{k}_{0}\right)$ in a factorized form of 2-point $S^{(2)}(\mathbf{k})$-s with necessarily positive values, we have found that in the domain of small wavenumbers, $S^{(3)}$ assumes negative values both in the experiment and in the simulations. What is, then, the significance of these negative values?

- First, in nonlinear scattering experiments, the 3-point function represents the lowest order nonlinearity and the existence of a negative domain should affect the resulting angular pattern, even though it is not easy to predict without further study the precise nature of this change.

- Second, the existence of the negative domain in the $\left(\mathbf{k}_{1}\right.$, $\mathbf{k}_{2}$ ) space also implies negative values of the quadratic density response function via the quadratic FluctuationDissipation Theorem. Thus, the response of physical systems to external potential perturbations will deviate here from that predicted by the Convolution Approximation. This is expected to become prominent under the effect of strong perturbations, where higher order response functions are applicable.

- Third, a further consequence of the negative values of $S^{(3)}$ is that negative values of the $h^{(3)}$ triplet correlation function may appear. Such a change in $h^{(3)}$ may have an effect on the thermodynamic properties of the system.

More detailed investigation of these effects warrants future work.

\section{ACKNOWLEDGMENTS}

We thank L. Pusztai and I. Tkachenko for useful discussions. This work has been supported by the Hungarian National Office for Research, Development and Innovation, via Grant Nos. NKFIH K-119357, NN-103150, and K115805, the J. Bolyai Research Scholarship of the Hungarian Academy of Sciences (PH) and the NSF.

\footnotetext{
${ }^{1}$ N. K. Ailawadi, Phys. Rep. 57, 241 (1980).

${ }^{2}$ A. Haymet, J. Phys. Colloq. 46, C9-27 (1985).

${ }^{3}$ U. Balucani and M. Zoppi, Dynamics of the Liquid State (Clarendon Press, 1995).
}

${ }^{4}$ K. I. Golden and J. T. Heath, Contrib. Plasma Phys. 55, 236 (2014).

${ }^{5}$ R. Kubo, J. Phys. Soc. Jpn. 12, 570 (1957).

${ }^{6}$ K. I. Golden, G. J. Kalman, and M. B. Silevitch, J. Stat. Phys. 6, 87 (1972).

${ }^{7}$ A. G. Sitenko, Zh. Eksp. Teor. Fiz. 75, 104 (1978) [Sov. Phys. JETP 48 51 (1978)].

${ }^{8}$ K. I. Golden and G. Kalman, Ann. Phys. 143, 160 (1982).

${ }^{9}$ P. Magyar, Z. Donkó, G. J. Kalman, and K. I. Golden, Phys. Rev. E 90, 023102 (2014)

${ }^{10}$ A. Ivlev, H. Löwen, G. Morfill, and C. P. Royall, Complex Plasmas and Colloidal Suspensions: Particle-Resolved Studies of Classical Liquids and Solids (World Scientific Publishing, 2012).

${ }^{11}$ P. M. Platzman, E. D. Isaacs, H. Williams, P. Zschack, and G. E. Ice, Phys. Rev. B 46, 12943 (1992).

${ }^{12}$ H. Sinn, F. Sette, U. Bergmann, C. Halcoussis, M. Krisch, R. Verbeni, and E. Burkel, Phys. Rev. Lett. 78, 1715 (1997).

${ }^{13}$ N. Maley, J. S. Lannin, and D. L. Price, Phys. Rev. Lett. 56, 1720 (1986).

${ }^{14}$ R. D. White, W. Tattersall, G. Boyle, R. E. Robson, S. Dujko, Z. Lj. Petrović, A. Banković, M. J. Brunger, J. P. Sullivan, S. J. Buckman, and G. Garcia, Appl. Radiat. Isot. 83, 77 (2014).

${ }^{15}$ S. Pothoczki, L. Temleitner, and L. Pusztai, J. Chem. Phys. 132, 164511 (2010).

${ }^{16}$ G. L. Hura, J. M. Sorenson, R. M. Glaeser, and T. Head-Gordon, J. Chem. Phys. 113, 9140 (2000).

${ }^{17}$ A. Hirata, P. Guan, T. Fujita, Y. Hirotsu, A. Inoue, A. R. Yavari, T. Sakurai, and M. Chen, Nat. Mater. 10, 28 (2011).

${ }^{18}$ Q. Ma, W. Zhou, D. E. Sayers, and M. A. Paesler, Phys. Rev. B 52, 10025 (1995).

${ }^{19}$ R. Thiele, T. Bornath, C. Fortmann, A. Höll, R. Redmer, H. Reinholz, G. Röpke, A. Wierling, S. H. Glenzer, and G. Gregori, Phys. Rev. E 78 026411 (2008).

${ }^{20}$ R. Thiele, P. Sperling, M. Chen, T. Bornath, R. R. Fäustlin, C. Fortmann, S. H. Glenzer, W.-D. Kraeft, A. Pukhov, S. Toleikis, T. Tschentscher, and R. Redmer, Phys. Rev. E 82, 056404 (2010).

${ }^{21}$ M. S. Murillo, Phys. Rev. E 81, 036403 (2010).

${ }^{22}$ A. N. Souza, D. J. Perkins, C. E. Starrett, D. Saumon, and S. B. Hansen, Phys. Rev. E 89, 023108 (2014).

${ }^{23}$ D. Riley, J. J. Angulo Gareta, A. Benuzzi-Mounaix, M. Esposito, E. Garcia Saiz, C. Gregory, F. Y. Khattak, M. Koenig, M. J. Lamb, and D. McSherry, Plasma Phys. Controlled Fusion 47, B491 (2005).

${ }^{24}$ P. A. Egelstaff, D. I. Piage, and C. R. T. Heard, Phys. Lett. A 30, 376 (1969).

${ }^{25}$ Y. Uehara, Y. T. Lee, T. Ree, and F. H. Ree, J. Chem. Phys. 70, 1884 (1979).

${ }^{26}$ D. Dhabal, M. Singh, K. T. Wikfeldt, and C. Chakravarty, J. Chem. Phys. 141, 174504 (2014).

${ }^{27}$ D. Coslovich, J. Chem. Phys. 138, 12A539 (2013).

${ }^{28}$ L. M. Sesé, J. Phys. Chem. B 112, 10241 (2008).

${ }^{29}$ V. D. Grouba, A. V. Zorin, and L. A. Sevastianov, Int. J. Mod. Phys. B 18 1 (2004).

${ }^{30}$ K. Zahn, G. Maret, C. Russ, and H. H. von Grunberg, Phys. Rev. Lett. 91, 115502 (2003).

${ }^{31}$ M. Born and H. S. Green, Proc. R. Soc. London, Ser. A 188, 10 (1946).

${ }^{32}$ O. S. Vaulina, O. F. Petrov, V. E. Fortov, A. V. Chernyshev, A. V. Gavrikov, and O. A. Shakhova, Phys. Rev. Lett. 93, 035004 (2004).

${ }^{33}$ V. E. Fortov, O. F. Petrov, and O. S. Vaulina, Phys. Rev. Lett. 101, 195003 (2008)

${ }^{34}$ O. S. Vaulina, I. E. Drangevski, X. G. Adamovich, O. F. Petrov, and V. E. Fortov, Phys. Rev. Lett. 97, 195001 (2006).

${ }^{35} \mathrm{H}$. Thomsen, P. Ludwig, M. Bonitz, and G. Kalman, in Abstracts of Strongly Coupled Coulomb Systems (SCCS) Conference, Budapest, Hungary (2011).

${ }^{36}$ H. W. Jackson and E. Feenberg, Rev. Mod. Phys. 34, 686 (1962).

${ }^{37}$ T. O'Neil and N. Rostoker, Phys. Fluids 8, 1109 (1965).

${ }^{38}$ T. J. Lie and Y. H. Ichikawa, Rev. Mod. Phys. 38, 680 (1966).

${ }^{39}$ H. Iyetomi and S. Ichimaru, Phys. Rev. A 27, 3241 (1983).

${ }^{40}$ P. Magyar, Z. Donkó, G. J. Kalman, and K. I. Golden, Contrib. Plasma Phys. 56, 816 (2016)

${ }^{41}$ J. L. Barrat, J. P. Hansen, and G. Pastore, Mol. Phys. 63, 747 (1988).

${ }^{42}$ H. Thomas, G. E. Morfill, V. Demmel, J. Goree, B. Feuerbacher, and D. Möhlmann, Phys. Rev. Lett. 73, 652 (1994); J. H. Chu and I. Lin, ibid. 72 4009 (1994); R. A. Quinn, C. Cui, J. Goree, J. B. Pieper, H. Thomas, and G. E. Morfill, Phys. Rev. E 53, R2049 (1996). 
${ }^{43}$ P. Hartmann, M. C. Sándor, A. Kovács, and Z. Donkó, Phys. Rev. E 84, 016404 (2011).

${ }^{44}$ B. Liu, J. Goree, and Y. Feng, Phys. Rev. Lett. 105, 085004 (2010).

${ }^{45}$ F. M. Peeters and X. Wu, Phys. Rev. A 35, 3109 (1987).

${ }^{46}$ G. J. Kalman, P. Hartmann, Z. Donkó, and M. Rosenberg, Phys. Rev. Lett. 92, 065001 (2004).

${ }^{47}$ Y. Feng, J. Goree, and B. Liu, Rev. Sci. Instrum. 78, 053704 (2007).

${ }^{48}$ Z. Donkó, G. J. Kalman, and P. Hartmann, J. Phys.: Condens. Matter 20, 413101 (2008).
${ }^{49}$ M. S. Murillo, Phys. Plasmas 7, 33 (2000); S. Nunomura, D. Samsonov, and J. Goree, Phys. Rev. Lett. 84, 5141 (2000); H. Ohta and S. Hamaguchi, ibid. 84, 6026 (2000); S. Zhdanov, S. Nunomura, D. Samsonov, and G. Morfill, Phys. Rev. E 68, 035401(R) (2003); A. Melzer, ibid. 67, 016411 (2003).

${ }^{50}$ V. E. Fortov et al., Phys. Rev. Lett. 90, 245005 (2003); P. Hartmann, G. J. Kalman, Z. Donkó, and K. Kutasi, Phys. Rev. E 72, 026409 (2005); T. Ott and M. Bonitz, Contrib. Plasma Phys. 55, 243-253 (2015) 